\title{
A Method of Calculating Bound States in a Unified Field Model
}

\author{
D. Großer, B. Hailer, L. Hornung, T. Lauxmann, and H. Stumpf \\ Institut für Theoretische Physik, Universität Tübingen
}

Z. Naturforsch. 38 a, 1056- 1063 (1983); received May 14, 1983

\begin{abstract}
In a model in which the usual elementary particles (leptons, quarks, photons, weak bosons, gluons, and so on) are bound states of truly elementary fermions we present a method for the calculation of the masses of these bound states. The kinetic energy of this model contains derivatives of second order so that the four-fermion interaction becomes renormalizable. The method uses explicit representations for the Hilbert space vectors of bound states.
\end{abstract}

\section{Introduction}

The standard $\mathrm{SU}(3) \times \mathrm{SU}(2) \times \mathrm{U}(1)$ model describes the interaction of particles in a way which is in agreement with present day experiments. In particular it seems that one of the outstanding predictions of this model, the $\mathrm{W}$ boson, has been found recently [1]. Nevertheless, there are several reasons why one would not like to consider this model as the fundamental theory of elementary particles:

(1) It contains more than twenty arbitrary parameters.

(2) There are at least 37 elementary particles which have different Lorentz properties: scalar bosons, spin $\frac{1}{2}$ fermions, and vector bosons.

(3) The model does not explain the fact that leptons and quarks form at least three families which have identical structure but distinct mass scales.

(4) There is no explanation of the fact that the electric charges of leptons and quarks obey simple relations.

At present there exist two ideas how to construct models which are more satisfactory with respect to these points. The first idea (called grand unification) consists in replacing the gauge group by a simple group which contains $\mathrm{SU}(3) \times \mathrm{SU}(2) \times \mathrm{U}(1)$ as a subgroup [2]. This reduces the number of gauge coupling constants from three to one. That means that the number of all arbitrary parameters is only slightly reduced. Since electric charge is typically a traceless generator of the simple gauge group the observed relations between lepton and quark charges are explained. Thus point (4) is understood.

Reprint requests to Prof. Dr. H. Stumpf, Institut für Theoretische Physik der Universität Tübingen, D-7400 Tübingen.
To spontaneously break the simple group down to $\mathrm{SU}(3) \times \mathrm{SU}(2) \times \mathrm{U}(1)$ additional scalar bosons have to be introduced. Therefore there is nearly no improvement with respect to points (1)-(3). On the other hand, a new problem (the hierarchy problem) arises from the fact that these models contain vector bosons with masses which belong to two widely different mass scales: $100 \mathrm{GeV}$ and $10^{15} \mathrm{GeV}$. This huge difference in mass scale can be described at tree level by fine tuning of the parameters of the model. It is, however, not stable under renormalization [3]. Even if one invokes supersymmetry [4] one can only achieve that this mass difference becomes stable but one cannot explain the difference itself.

The second idea assumes that at least some of the elementary particles in the standard model are not truly elementary, but are bound states of more fundamental constituents [5]. Such models could lead to an improvement with respect to points (1)-(4). Among the existing models the one proposed by Harari and Shupe [6] stands out because of its simplicity. In this model leptons and quarks are bound states of two basic spin $\frac{1}{2}$ fermions called rishons by Harari. Because electrons have electric charge and neutrinos are neutral one needs at least two constituents, one with charge and another one without. Thus in this respect the model is minimal. It explains point (4) in a very simple and natural way. The question of the interaction of the rishons, however, was left open.

Later on, Harari and Seiberg [7] in order to introduce interaction, supplied rishons with colour and hypercolour charges. The hypercolour forces are supposed to bind together rishons into bound states which are hypercolour neutral: leptons and quarks. These hypercolour forces become strong so rapidly with growing distance that leptons and 
quarks are sufficiently small. This model contains only three coupling constants. On the other hand, it assumes 18 fundamental fermions of spin $\frac{1}{2}$ and 17 fundamental vector bosons. So the number of basic particles is no longer small and they have two different spins: $\frac{1}{2}$ and 1 . Simplicity is lost.

The strong interaction between nucleons can be described as being mediated by pions, as was suggested by Yukawa. Gell-Mann [8] described the strong interactions within the framework of a nonAbelian gauge theory and the observed vector mesons served as gauge bosons. Today we know that not only the nucleons but also the exchanged bosons are composite particles. In analogy to this one might speculate that not only the leptons and quarks but also the vector bosons which mediate their interaction, the gluons, the weak bosons, the photon, etc., are composites.

In a previous publication [9] two of the present authors proposed a model which contains only the two original rishons as basic particles. An immediate difficulty in such an approach is the fact that already the most simple Lorentz invariant interaction is a product of four rishon fields and hence is non-renormalizable if the kinetic part of the Lagrangian contains only derivatives of first order. To obtain renormalizability we introduced derivatives of second order. Field theories with higher derivatives were considered by Bopp, Podolski, Pais, and Uhlenbeck, and Wildermuth [10]. They have recently been advocated by one of the present authors [11]. Higher derivatives appear in conformal gravity [12] and conformal supergravity [13]. Higher derivatives lead to negative-norm states in Fock space and hence the S-Matrix is not unitary at tree level. There are, however, possible solutions to this problem. Lee and Wick [14] have shown that loop corrections can push the ghost poles off the real axis into the complex plane. If one then defines the physical subspace as being spanned by those eigenvectors of the Hamiltonian which belong to real eigenvalues, then the S-matrix will be unitary. In addition to this, non-perturbative effects may distort the theory in such a way that the S-matrix is unitary at least in a suitable subspace. This has been discussed by one of the present authors [15] and will be further discussed in a forthcoming publication [16] by the same author. Let us assume for the time being that this problem can be solved in a satisfactory way.
In this model scale invariance of the Lagrangian is broken only by parameters with dimension of mass. The four-fermion interaction is required to be invariant under space inversion $P$ and charge conjugation $C$. $P$ and $C$ invariance is then broken by terms of dimension 3. Therefore, as has been shown by Symanzik [17], they induce no counterterms of dimension 4 . Symmetry breaking contributions e.g. to a four-point function are therefore finite and hence small for appropriate adjustment of parameters. Remarkably all terms of dimension 3 are invariant under $C P$. $C P$ can be broken by terms of dimension 2. Repeating the above argument we conclude that we can keep $C P$ violation relatively small by adjusting parameters. Therefore the model allows in a natural way to describe the observed hierarchy of $P, C$, and $C P$ violation.

There is an additional advantage which a model with a fermion selfinteraction may have over a nonabelian gauge theory. Leptons and quarks are very small $\left(\lesssim 10^{-16} \mathrm{~cm}\right)$ and therefore due to the uncertainty principle the kinetic energy of the constituents is $\gtrsim 100 \mathrm{GeV}$. Lepton and quark masses are nearly zero on that scale. To reproduce this fact in the theory we need a great amount of binding energy. Non-abelian gauge theories are asymptotically free which means that the interaction becomes small if the interacting particles come close together. Therefore it is not at all clear whether a non-abelian gauge theory will provide enough binding energy and one may be better off with a fermion selfinteraction which is especially effective if the particles are close together. There is no such problem in QCD because the kinetic energy of the quarks is of the same order of magnitude as the mass of the nucleon.

In the present publication we want to study this model in further detail. In Section II we introduce auxiliary fields and quantize the theory in terms of these fields in accordance with Poincare invariance. In Section III we represent the Hilbert space vectors by functionals which are constructed from the vectors and the fields and derive equations for these functionals starting from the equations of motion and the commutation relations. These equations contain derivatives with respect to the space-time coordinates. For eigenvectors of energy-momentum we eliminate in Section IV the time derivative with the help of four-dimensional translation invariance using a method which has been applied before [18] 
in a quantum mechanical model of field theory. We obtain a functional Schrödinger equation which contains the mass of the state and therefore in principle enables one to calculate masses. Since the rigorous solution of functional equations is not possible at the time being we approximate in Section $\mathrm{V}$ the functional in analogy to the New Tamm-Dancoff method [19] by setting equal to zero all functions which are generated by the functional and depend on more than $n$ variables. The physical idea behind this approach is the assumption that functions with more than $n_{0}$ variables ( $n_{0}$ depending on the particular bound state under consideration) describe contributions of constituent-anticonstituent pairs and that the importance of these contributions is decreasing with increasing number of pairs. The resulting approximation scheme is a systematic nonperturbative calculation procedure for the masses of bound states. The application of this approximation scheme to nonrelativistic many-body problems has been suggested by Bleuler et al. [20].

If the idea of composite leptons and quarks is correct it opens a possibility to understand the lepton and quark mass spectrum. But this hope can of course only be realized if we are able to calculate bound state masses. Therefore a systematic nonperturbative calculation procedure is of utmost importance. The usual methods are: the bag-model [21], lattice calculations [22], and the Bethe-Salpeter equation [23]. The bag model treats constituents relativistically and is phenomenologically quite successful. It is, however, not a satisfactory solution of the problem because it relies on boundary conditions which are introduced ad hoc. Considerable effort has been invested into lattice calculations and they produce quite reasonable numbers. These calculations, however, have been strongly critisized by Hasenfratz and Montvay [24]. The BetheSalpeter Equation, apart from the fact that it has solutions with negative norm even in theories in which no indefinite metric is expected (Nakanishi [25]), needs perturbative input for the BetheSalpeter kernel. The solution in a sense sums up a class of Feynman diagrams containing diagrams of arbitrarily high order in the coupling constant and therefore obtains bound states. However, by using perturbative approximations for the kernel one assumes that the theory is analytic in the coupling constant and by not summing all Feynman diagrams one assumes that the omitted ones are not relevant.
Both assumptions may very well be wrong. Therefore we think that our approximation scheme deserves investigation.

At the end of this introduction we want to mention some related approaches and to point out the differences. Terazawa and Akama [26] have considered models in which all elementary particles are spin $\frac{1}{2}$ fermions. They admit only first order derivatives and therefore have to introduce a cutoff. Amati and Venziano [27] also start with fundamental fermionic fields only. They choose a nonpolynomial interaction so that some symmetries become local symmetries. The approach of Dürr [28] (originally due to Heisenberg [29]) again starts with a basic fermion field. This fermion field, however, is not directly connected with particles. The fields which are connected with particles are products of this "urfield". So to describe e.g. a boson, Dürr would construct a boson field operator from the urfield and would try to understand the properties of the boson from the properties of that boson field, whereas we would like to think of the boson as being a bound state of fermionic constituents.

\section{The model and its quantization}

Our model contains two basic Dirac spinors: $T(x)$ (carrying $1 / 3$ of the charge of the positron) and $V(x)$ (neutral). The associated particles are collectively called rishons. The Lagrangian is assumed to be a sum of a kinetic term and an interaction term:

$$
\mathscr{f}=\mathscr{f}_{\text {kin }}+\mathscr{f}_{\mathrm{I}} .
$$

For $\mathcal{L}_{\text {kin }}$ we take the most general expression which is hermitean, Lorentz invariant, bilinear in the fields, contains derivatives up to second order, and conserves charge and rishon number (= number of $T$ particles plus number of $V$ particles):

$$
\begin{aligned}
f_{\mathrm{kin}}= & f_{2}+f_{1}+f_{0}, \\
\mathscr{f}_{2}= & \left(\partial_{\mu} \bar{T}\right) \partial^{\mu} T+(T \rightarrow V), \\
\mathscr{f}_{1}= & i M_{\mathrm{T} 1} \bar{T} \gamma^{\mu} \frac{1-\gamma_{5}}{2} \partial_{\mu} T \\
& +i M_{\mathrm{T} 2} \bar{T} \gamma^{\mu} \frac{1+\gamma_{5}}{2} \partial_{\mu} T+(T \rightarrow V), \\
\mathscr{f}_{0}= & \mu_{\mathrm{T}}^{2} \bar{T} \frac{1-\gamma_{5}}{2} T \\
& +\mu_{\mathrm{T}}^{2 *} \bar{T} \frac{1+\gamma_{5}}{2} T+(T \rightarrow V),
\end{aligned}
$$


where $M_{\mathrm{Ti}}$ and $M_{\mathrm{Vi}}$ are real. For $\mathscr{f}_{\mathrm{I}}$ we take the most general four-fermion interaction which is hermitean, invariant under Lorentz transformations, $\mathrm{P}$ and $\mathrm{C}$, and conserves charge and rishon number:

$$
\begin{aligned}
\mathscr{f}_{\mathrm{I}}= & f_{1} \bar{T} T \bar{T} T+f_{2} \bar{T} \gamma_{\mu} T \bar{T} \gamma^{\mu} T \\
& +f_{3} \bar{T} \gamma_{\mu} \gamma_{5} T \bar{T} \gamma^{\mu} \gamma_{5} T+\left(T \rightarrow V, f_{i} \rightarrow g_{i}\right) \\
& +h_{1} \bar{T} T \bar{V} V+h_{2} \bar{T} i \gamma_{5} T \bar{V} i \gamma_{5} V \\
& +h_{3} \bar{T} \gamma_{\mu} T \bar{V} \gamma^{\mu} V+h_{4} \bar{T} \gamma_{\mu} \gamma_{5} T \bar{V} \gamma^{\mu} \gamma_{5} V \\
& +h_{5} \bar{T} \sigma_{\mu \nu} T \bar{V} \sigma^{\mu \nu} V
\end{aligned}
$$

where the coupling constants are real. The number of independent coupling constants is somewhat high. But since we have no convincing reason for setting some of them equal to zero we prefer to keep them all at this point.

Since in nature $\mathrm{P}$ and $\mathrm{C}$ violations are restricted to weak interactions we will facilitate our calculations in the beginning by assuming that the Lagrangian is invariant under $\mathrm{P}$ and $\mathrm{C}$. This implies

$$
\begin{aligned}
& M_{\mathrm{T} 1}=M_{\mathrm{T} 2}, \quad M_{\mathrm{V} 1}=M_{\mathrm{V} 2}, \\
& \mu_{\mathrm{T}}^{2^{*}}=\mu_{\mathrm{T}}^{2}, \quad \mu_{\mathrm{V}}^{2 *}=\mu_{\mathrm{V}}^{2} .
\end{aligned}
$$

In this case we may write

$$
\mathscr{f}_{\text {kin }}=\bar{T}\left(i \not \supset-m_{\mathrm{T} 1}\right)\left(i \not \supset-m_{\mathrm{T} 2}\right) T+(T \rightarrow V)
$$

if

$$
\begin{array}{ll}
M_{\mathrm{T} 1}=-\left(m_{\mathrm{T} 1}+m_{\mathrm{T} 2}\right) ; & \mu_{\mathrm{T}}^{2}=m_{\mathrm{T} 1} m_{\mathrm{T} 2} ; \\
M_{\mathrm{V} 1}=-\left(m_{\mathrm{V} 1}+m_{\mathrm{V} 2}\right) ; & \mu_{\mathrm{V}}^{2}=m_{\mathrm{V} 1} m_{\mathrm{V} 2} .
\end{array}
$$

If we set

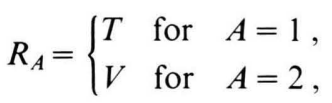

and in analogy to this

$$
m_{A i}=\left\{\begin{array}{lll}
m_{T i} & \text { for } & A=1, \\
m_{V i} & \text { for } & A=2,
\end{array}\right.
$$

then one has

$$
\mathcal{f}_{\text {kin }}=\sum_{A=1}^{2} \overline{R_{A}}\left(i \not \varnothing-m_{A 1}\right)\left(i \not \supset-m_{A 2}\right) R_{A} .
$$

Now in order to quantize the model we introduce auxiliary fields

$$
R_{A i}=\prod_{\substack{k=1 \\ k \neq i}}^{2}\left(i \nexists-m_{A k}\right) R_{A} .
$$

If we define

$$
x_{A i}=\prod_{\substack{k=1 \\ k \neq i}}^{2}\left(m_{A i}-m_{A k}\right)^{-1}
$$

then one has

$$
R_{A}=\sum_{i=1}^{2} x_{A i} R_{A i} .
$$

These auxiliary fields and the decomposition (2.12) were introduced by Pais and Uhlenbeck [10]. As far as interactions are concerned they consider only the usual coupling to the electromagnetic field. They claim that the Lagrangian, when expressed in terms of the auxiliary fields, is not equivalent to the original Lagrangian, i.e. leads to different field equations, and therefore conclude that the introduction of the auxiliary fields is of no use. Recently, one of the present authors [30] arrived independently at the decomposition (2.12) and found that a higher order derivative field equation for a spinor field with self-interaction $\left(R_{A}\right.$ in our present model $)$ is equivalent to first order equations for the auxiliary fields. The fact that we have higher order equations for $R_{A}$ but first order equations for $R_{A i}$ is of crucial importance in the following.

For convenience we absorb the factor $x_{A i}$ into the fields by defining

$$
S_{A i}=x_{A i} R_{A i} .
$$

When $\mathscr{f}_{\text {kin }}$ is expressed in terms of these fields one finds

$$
f_{\text {kin }}=\sum_{A=1}^{2} \sum_{i=1}^{2} \frac{1}{x_{A i}} \overline{S_{A i}}\left(i \not D-m_{A i}\right) S_{A i}
$$

and for $\mathscr{f}_{\mathrm{I}}$ one obtains

$$
\begin{aligned}
\mathscr{f}_{\mathrm{I}}= & \sum_{l=1}^{2} \sum_{k=1}^{2} \sum_{j=1}^{2} \sum_{i=1}^{2} V_{A B \alpha \beta \gamma \delta} \overline{S_{A i \alpha}} \\
& \cdot \overline{S_{B j \beta}} S_{A k \gamma} S_{B l \delta},
\end{aligned}
$$

where

$$
V_{A B \alpha \beta \gamma \delta}=F_{A \alpha \beta \gamma \delta} \delta_{A B}+H_{\alpha \beta \gamma \delta} \delta_{A 1} \delta_{B 2}
$$

and

$$
\begin{aligned}
F_{1 \alpha \beta \gamma \delta}= & -f_{1} \delta_{\alpha \gamma} \delta_{\beta \delta}-f_{2} \gamma_{\mu \alpha \gamma} \gamma_{\beta \delta}^{\mu} \\
& -f_{3}\left(\gamma_{\mu} \gamma_{5}\right)_{\alpha \gamma}\left(\gamma^{\mu} \gamma_{5}\right)_{\beta \delta}, \\
F_{2 \alpha \beta \gamma \delta}= & -g_{1} \delta_{\alpha \gamma} \delta_{\beta \delta}-g_{2} \gamma_{\mu \alpha \gamma} \gamma_{\beta \delta}^{\mu} \\
& -g_{3}\left(\gamma_{\mu} \gamma_{5}\right)_{\alpha \gamma}\left(\gamma^{\mu} \gamma_{5}\right)_{\beta \delta},
\end{aligned}
$$




$$
\begin{aligned}
H_{\alpha \beta \gamma \delta}= & -h_{1} \delta_{\alpha \gamma} \delta_{\beta \delta}-h_{2}\left(i \gamma_{5}\right)_{\alpha \gamma}\left(i \gamma_{5}\right)_{\beta \delta} \\
& -h_{3} \gamma_{\mu \alpha \gamma} \gamma_{\beta \delta}^{\mu}-h_{4}\left(\gamma_{\mu} \gamma_{5}\right)_{\alpha \gamma}\left(\gamma^{\mu} \gamma_{5}\right)_{\beta \delta} \\
& -h_{5} \sigma_{\mu v \alpha \gamma} \sigma_{\beta \delta}^{\mu \nu} .
\end{aligned}
$$

The minus sign in $(2.17)-(2.19)$ is due to the fact that in going from (2.3) to (2.15) we have pushed all adjoint spinors to the left (we are still at the classical level and our fields are anticommuting c-numbers).

We now want to quantize the model. As is well known, the usual canonical procedure does not work due to the fact that the Lagrangian is of first order in time derivatives. In going to the Hamiltonian formalism one defines momenta conjugate to the field variables $S_{A i \alpha}$ by

$$
\Pi_{A i \alpha}=\partial f / \partial \partial_{0} S_{A i \alpha} .
$$

This equation is then used to eliminate time derivatives of the field variables. But since $\mathscr{f}$ is of first order in the time derivatives the right-hand side of (2.20) is independent of $\partial_{0} S_{\text {Aix }}$. Hence the elimination is not possible. One can nevertheless postulate

$$
\begin{aligned}
& \left\{\Pi_{A i \alpha}(t, \vec{x}), S_{A^{\prime} i^{\prime} \alpha^{\prime}}\left(t, \vec{x}^{\prime}\right)\right\} \\
& \quad=-i \delta_{A A^{\prime}} \delta_{i i^{\prime}} \delta_{\alpha \alpha^{\prime}} \delta\left(\vec{x}-\vec{x}^{\prime}\right) .
\end{aligned}
$$

But this then needs further justification. Before giving this, let us consider the consequences of (2.21). Evaluating (2.20) and inserting into (2.21) yields

$$
\begin{aligned}
& \left\{S_{A i \alpha}^{+}(t, \vec{x}), S_{A^{\prime} i^{\prime} \alpha^{\prime}}\left(t, \vec{x}^{\prime}\right)\right\} \\
& \quad=x_{A i} \delta_{A A^{\prime}} \delta_{i i^{\prime}} \delta_{\alpha \alpha^{\prime}} \delta\left(\vec{x}-\vec{x}^{\prime}\right) .
\end{aligned}
$$

From the definition of $x_{A i},(2.11)$, it follows that either $x_{A 1}$ or $x_{A 2}$ is negative. Hence by taking the expectation value of (2.22) with respect to an arbitrary vector of Hilbert space one finds immediately that the Hilbert space contains vectors of negative norm.

Invariance under translations implies by Noether's theorem four conserved quantities

$P_{v}=\int \mathrm{d}^{3} x\left(\sum_{A=1}^{2} \sum_{i=1}^{2} i \frac{1}{x_{A i}} \overline{S_{A i}} \gamma_{0} \partial_{v} S_{A i}-\delta_{0 v} \mathcal{f}\right)$.

In Hilbert space the translations are generated by four operators $\overline{P_{v}}$ for which one has

$$
\left[\overline{P_{v}}, S_{A i}\right]=-i \partial_{v} S_{A i}
$$

Now we require that the commutation relations together with the equation of motion

$$
\begin{aligned}
& \left(i \gamma_{\alpha \beta}^{\mu} \partial_{\mu}-m_{A i} \delta_{\alpha \beta}\right) S_{A i \beta} \\
& =x_{A i}\left(V_{A B \alpha \beta \gamma \delta}+V_{B A \beta \alpha \delta \gamma}\right) \overline{S_{B j \beta}} S_{B l \delta} S_{A k \gamma}
\end{aligned}
$$

imply $P_{v}=\overline{P_{v}}$. One can check by direct calculation that this is in fact true if the commutation relations (2.22) are adopted.

From the definition of $x_{A i}$ it follows that $x_{A 2}=-x_{A 1}$. Hence the commutation relations for the auxiliary fields imply for the original fields

$$
\left\{R_{A \alpha}^{+}(t, \vec{x}), R_{A^{\prime} \alpha^{\prime}}\left(t, \vec{x}^{\prime}\right)\right\}=0 .
$$

Due to $x_{A 1}+x_{A 2}=0$ the singularities of the two point Green's function of the field $R_{A}$ on the light cone, which are independent of mass, cancel each other (in the same way as in the Pauli-Villars regularization). Hence the singularities of this Green's function are less severe.

\section{Representations of states}

To simplify our notation we define

$$
\psi_{A i \Lambda \alpha}(x)=\left\{\begin{array}{lll}
S_{A i \alpha}(x) & \text { for } & \Lambda=1, \\
S_{A i \alpha}(x) & \text { for } & \Lambda=2,
\end{array}\right.
$$

and write

$$
\psi_{A i \Lambda x}(x) \equiv \psi_{Z}(x) \equiv \psi_{I} .
$$

We then characterize an arbitrary state $|a\rangle$ by the amplitudes

$$
\left\langle 0\left|T \psi_{I_{1}} \ldots \psi_{I_{n}}\right| a\right\rangle, \quad n=0,1,2, \ldots
$$

If we introduce the functional

$T(j, a)=\sum_{n=0}^{\infty} \frac{i^{n}}{n !} \cdot \sum_{I_{1} \ldots I_{n}}\left\langle 0\left|T \psi_{I_{1}} \ldots \psi_{I_{n}}\right| a\right\rangle j_{I_{1}} \ldots j_{I_{n}}$,

where the $j_{I}$ are generators of an infinite-dimensional Grassmann algebra, then one can regain the amplitudes (3.3) by functional differentiation:

$$
\begin{gathered}
\left.\frac{1}{i} \frac{\delta}{\delta j_{I_{n}}} \ldots \frac{1}{i} \frac{\delta}{\delta j_{I_{1}}} T(j, a)\right|_{j=0} \\
=\left\langle 0\left|T \psi_{I_{1}} \ldots \psi_{I_{n}}\right| a\right\rangle .
\end{gathered}
$$

and hence the functional $T(j, a)$ represents the state $a)$. There is one remark in order here. The lefthand side of (3.5) is antisymmetric with respect to 
the indices $I_{i}$. Hence (3.5) can only be correct if the amplitudes (3.3) are also antisymmetric. In forming $T(j, a)$ and differentiating non-antisymmetric parts of the amplitudes would be lost. Now if the time labels belonging to two indices $I_{i}$ and $I_{k}$ are different then the time ordering guarantees that the amplitude is antisymmetric with respect to $I_{i}$ and $I_{k}$. On the other hand, if these time labels are equal then (3.3) is not yet defined and we are still free to define it in such a way that the amplitude becomes antisymmetric. Let us consider the following example: $n=5$ and $t_{I_{1}}>t_{I_{2}}=t_{I_{3}}=t_{I_{4}}>t_{I_{5}}$. In this case we set

$$
\begin{aligned}
& T \psi_{I_{1}} \ldots \psi_{I_{5}} \\
& \quad=\psi_{I_{1}}\left(\frac{1}{3 !} \sum_{P} \operatorname{sgn}(P) \psi_{I_{P(2)}} \psi_{I_{P(3)}} \psi_{I_{P(4)}}\right) \psi_{I_{5}},
\end{aligned}
$$

where the sum is over all permutations of 2,3 , and 4.

The functional differentiation $\delta / \delta j_{I}$ is an operator in the Grassmann algebra and $j_{I}$ itself can be considered as an operator. These operators have the anticommunication relations

$$
\begin{aligned}
& \left\{j_{I_{1}}, j_{I_{2}}\right\}=\left\{\frac{\delta}{\delta j_{I_{1}}}, \frac{\delta}{\delta j_{I_{2}}}\right\}=0, \\
& \left\{j_{I_{1}}, \frac{\delta}{\delta j_{I_{2}}}\right\}=\delta_{I_{1} I_{2}} .
\end{aligned}
$$

Hence there is a Fock space representation of this Jordan-Wigner algebra characterized by

$$
\frac{\delta}{\delta j_{I}}|0\rangle=0 \text {. }
$$

Therefore we can associate with every element $T(j, a)$ of the Grassmann algebra a state in this Fock space

$$
\begin{aligned}
|T(j, a)\rangle= & \sum_{n=0}^{\infty} \frac{i^{n}}{n !} \\
& \cdot \sum_{I_{1} \ldots I_{n}}\left\langle 0\left|T \psi_{I_{1}} \ldots \psi_{I_{n}}\right| a\right\rangle j_{I_{1}} \ldots j_{I_{n}}|0\rangle .
\end{aligned}
$$

The equations of motion (2.25) and the commutation relations (2.22) now have the form

$$
\begin{gathered}
\sum_{Z_{2}} D_{Z_{1} Z_{2}}^{\mu} \partial_{\mu} \psi_{Z_{2}}-m_{Z_{1}} \psi_{Z_{1}} \\
=\sum_{Z_{2} Z_{3} Z_{4}} U_{Z_{1} Z_{2} Z_{3} Z_{4}} \psi_{Z_{2}} \psi_{Z_{3}} \psi_{Z_{4}}, \\
\left\{\psi_{Z_{1}}\left(t, \vec{x}_{1}\right), \psi_{Z_{2}}\left(t, \vec{x}_{2}\right)\right\}=A_{Z_{1} Z_{2}} \delta\left(\vec{x}_{1}-\vec{x}_{2}\right),
\end{gathered}
$$

where

$$
\begin{aligned}
& D_{Z_{1} Z_{2}}^{\mu} \equiv D_{A i \Lambda \times B j \Gamma \beta}^{\mu} \\
& =i \delta_{A B} \delta_{i j} \delta_{A \Gamma}\left\{\begin{array}{rll}
\gamma_{\alpha \beta}^{\mu} & \text { for } & \Lambda=1, \\
-\gamma_{\alpha \beta}^{\mu T} & \text { for } & \Lambda=2,
\end{array}\right. \\
& m_{Z_{1}} \equiv m_{A i \Lambda \alpha}=m_{A i}, \\
& U_{A B C D}=x_{A i} \delta_{B C} \delta_{A D} \delta_{2 \Gamma} \\
& i j k l \\
& \underset{\alpha \beta \gamma \delta}{1 \Gamma \Omega \Sigma} \quad \cdot \delta_{1 \Omega} \delta_{1 \Sigma}\left(V_{A B \alpha \beta \delta \gamma}+V_{B A \beta \alpha \gamma \delta}\right), \\
& U_{A B C D}=-x_{A i} \delta_{A C} \delta_{B D} \delta_{2 \Gamma} \delta_{2 \Omega}
\end{aligned}
$$

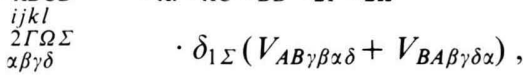

$$
\begin{aligned}
& A_{Z_{1} Z_{2}} \equiv A_{A i \Lambda \alpha B j \Gamma \beta}=\sigma_{1 A \Gamma} x_{A i} \delta_{A B} \delta_{i j} \gamma_{4 \alpha \beta} \text {, }
\end{aligned}
$$

and $\sigma_{1}$ is the first Pauli matrix.

If one applies to $|T(j, a)\rangle$ first the functional derivative

$$
\frac{1}{i} \frac{\delta}{\delta j_{Z}(x)} \equiv \frac{1}{i} \partial_{Z}(x)
$$

and then the differential operator $D_{Z_{1} Z_{2}}^{\mu} \partial_{\mu}$, then using the equations of motion (3.7) and the commutation relations (3.8) one finds

$$
\begin{aligned}
D_{Z_{1} Z_{2}}^{\mu} \partial_{\mu} & \frac{1}{i} \partial_{Z_{2}}(x)|T(j, a)\rangle-m_{Z_{1}} \frac{1}{i} \partial_{Z_{1}}(x)|T(j, a)\rangle \\
= & i D_{Z_{1} Z_{2}}^{0} A_{Z_{2} Z_{3}} j_{Z_{3}}(x)|T(j, a)\rangle \\
& +U_{Z_{1} Z_{2} Z_{3} Z_{4}} \frac{1}{i} \partial_{Z_{4}}(x) \frac{1}{i} \partial_{Z_{3}}(x) \frac{1}{i} \\
& \cdot \partial_{Z_{2}}(x)|T(j, a)\rangle .
\end{aligned}
$$

If we apply to this equation functional differentiation $n$ times $(n=0,1,2, \ldots)$ and put $j_{I}=0$ afterwards, then we obtain an infinite set of coupled equations for the amplitudes (3.3). The first term on the right-hand side of (3.15) introduces $\delta$-functions into this set of equations. This term can be eliminated by going over to a second functional

$|\Phi(j, a)\rangle=\exp \left\{\frac{1}{2} \sum_{I_{1} I_{2}} j_{I_{1}} F_{I_{1} I_{2}} j_{I_{2}}\right\}|T(j, a)\rangle$,

where

$$
\begin{aligned}
F_{I_{1} I_{2}} & \equiv F_{Z_{1} Z_{2}}\left(x_{1}, x_{2}\right) \\
& =\left\langle 0\left|T\left(\psi_{Z_{1}}\left(x_{1}\right) \psi_{Z_{2}}\left(x_{2}\right)\right)\right| 0\right\rangle
\end{aligned}
$$

is the propagator of the free theory. From the functional equation (3.15) for $|T(j, a)\rangle$ one finds for $\Phi(j, a)\rangle$ :

$$
\begin{aligned}
& D_{Z_{1} Z_{2}}^{\mu} \partial_{\mu} \partial_{Z_{2}}(x)|\Phi(j, a)\rangle-m_{Z_{1}} \partial_{Z_{1}}(x)|\Phi(j, a)\rangle \\
& \quad=-U_{Z_{1} Z_{2} Z_{3} Z_{4}} \mathrm{~d}_{Z_{4}}(x) \mathrm{d}_{Z_{3}}(x) \mathrm{d}_{Z_{2}}(x)|\Phi(j, a)\rangle,(3.18
\end{aligned}
$$


where

$\mathrm{d}_{Z}(x)=\partial_{Z}(x)-\sum_{Z_{1}} \int \mathrm{d}^{4} x_{1} F_{Z Z_{1}}\left(x, x_{1}\right) j_{Z_{1}}\left(x_{1}\right)$.

Equation (3.18) does not contain any more the dangerous term $A_{Z Z^{\prime}} j_{Z^{\prime}}(x)$.

\section{Eigenvalue equation and elimination of time derivatives}

Let us now assume that the state $|a\rangle$ is an eigenstate of $P_{\mu}$ :

$$
P_{\mu}|a\rangle=p_{\mu}|a\rangle .
$$

Using (2.24) one can then derive:

$$
\sum_{Z} \int \mathrm{d}^{4} x j_{Z}(x) i \partial_{\mu} \partial_{Z}(x)|T(j, a)\rangle=p_{\mu}|T(j, a)\rangle .
$$

The details of this derivation are contained in [31]. From the definition of $|\Phi(j, a)\rangle$ and the fact that $F_{I_{1} I_{2}}$ is a vacuum expectation value it follows that $\Phi(j, a)\rangle$ also satisfies

$\sum_{Z} \int \mathrm{d}^{4} x j_{Z}(x) i \partial_{\mu} \partial_{Z}(x)|\Phi(j, a)\rangle=p_{\mu}|\Phi(j, a)\rangle$.

Now (4.3) for $\mu=0$ and (3.18) are both linear in the time derivative and therefore this derivative can be
This is a functional eigenvalue equation which we cannot solve. We give in the next section an approximation scheme which reduces the problem to the solution of equations which are of the same type as the non-relativistic Schrödinger equation.

\section{Calculation of the Rishon-Antirishon Bound State}

The functional $\Phi(j, a)$ can be written in the following way:

$$
|\Phi(j, a)\rangle=\sum_{n=0}^{\infty} \frac{i^{n}}{n !} \sum_{I_{1} \ldots I_{n}} \varphi_{I_{1} \ldots I_{n}} j_{I_{1}} \ldots j_{I_{n}}|0\rangle .
$$

If $|a\rangle$ is a rishon-antirishon bound state then due to the conservation of the rishonnumber terms with odd $n$ are missing. If we insert (5.1) into (4.4) we obtain an infinite set of coupled equations for the $\varphi_{I_{1} \ldots I_{n}}$. Since we are not able to solve this infinite set of equations we approximate $\Phi(j, a)$ by

$$
|\Phi(j, a)\rangle=-\frac{1}{2} \sum_{I_{1} I_{2}} \varphi_{I_{1} I_{2}} j_{I_{1}} j_{I_{2}}|0\rangle .
$$

The physical idea of this approximation has been described in the introduction. Instead of inserting (5.2) into (4.4) we can apply $\partial_{\bar{z}_{1}}\left(\bar{x}_{1}\right) \partial_{\bar{z}_{2}}\left(\bar{x}_{2}\right)$ to both sides of (4.4) and then put $j=0$. The approximation (5.2) then consists in setting $\varphi_{I_{1} \ldots I_{n}}=0$ for $n>2$. One obtains

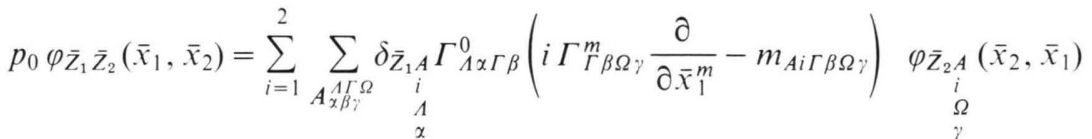

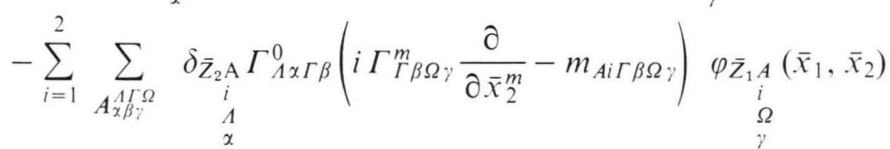

$$
\begin{aligned}
& +\sum_{y_{1} y_{2} y_{3}, y_{4}}\left(\delta_{\bar{Z}_{2 \nu_{1}}}-\delta_{\left.\bar{Z}_{2 \nu_{1}}\right)}\right) T_{y_{1} y_{2} y_{3} y_{4}} \sum_{\left(y_{2} y_{3} y_{4}\right)}\left(\sum_{i=1}^{2} F_{i} \bar{Z}_{1}\left(\bar{x}_{2}, \bar{x}_{1}\right)\right) \sum_{j, k=1}^{2} \varphi_{\substack{j k \\
y_{3} y_{4}}}\left(\bar{x}_{2}, \bar{x}_{2}\right) \\
& -\sum_{y_{1} y_{2} y_{3} y_{4}}\left(\delta_{\bar{Z}_{1} y_{1}}^{1}-\delta_{\bar{Z}_{1} y_{1}}^{2}\right) T_{y_{1} y_{2} y_{3} y_{4}} \sum_{\left(y_{2} y_{3} y_{4}\right)}\left(\sum_{i=1}^{2} F_{i} \bar{Z}_{2}\left(\bar{x}_{1}, \bar{x}_{2}\right)\right) \sum_{j, k=1}^{2} \varphi_{j} \varphi_{y_{3} y_{4}}\left(\bar{x}_{1}, \bar{x}_{1}\right),
\end{aligned}
$$

eliminated. One obtains:

$$
\begin{aligned}
p_{0} \Phi & \Phi, a)\rangle \\
= & \sum_{Z Z_{1} Z_{2}} \int \mathrm{d}^{4} x j_{Z}(x) i D_{Z Z_{1}}^{0}\left(D_{Z_{1} Z_{2}}^{m} \partial_{m}-m_{Z_{1}} \delta_{Z_{1} Z_{2}}\right) \\
& \left.\cdot \partial_{Z_{2}}(x) \Phi(j, a)\right\rangle \\
& +\sum_{Z Z_{1} Z_{2} Z_{3} Z_{4}} \int \mathrm{d}^{4} x j_{Z}(x) i D_{Z Z_{1}}^{0} U_{Z_{1} Z_{2} Z_{3} Z_{4}} \mathrm{~d}_{Z_{4}}(x) \\
& \cdot d_{Z_{3}}(x) d_{Z_{2}}(x)|\Phi(j, a)\rangle .
\end{aligned}
$$

where we have in some places resolved the multiindex $Z$ into

$$
Z \equiv A i \Lambda \alpha \equiv i y
$$

such that $y \equiv A \Lambda \alpha$ and where

$$
\begin{aligned}
& \varphi_{Z_{1} Z_{2}}\left(x_{1}, x_{2}\right)=\varphi_{I_{1} I_{2}}, \\
& \Gamma_{A \alpha \Gamma \beta}^{\mu}=\delta_{A \Gamma}\left\{\begin{array}{r}
\gamma_{\alpha \beta}^{\mu} \text { for } \Lambda=1, \\
-\gamma_{\alpha \beta}^{\mu T} \text { for } \Lambda=2,
\end{array}\right.
\end{aligned}
$$




$$
\begin{aligned}
& m_{A i \Gamma \beta \Omega \gamma}=m_{A i} \delta_{\Gamma \Omega} \delta_{\beta \gamma}, \\
& T_{\substack{A_{1} A_{2} A_{3} A_{4} \\
A_{1} \Lambda_{2} \Lambda_{3} \Lambda_{4} \\
\alpha_{1} \alpha_{2} \alpha_{3} \alpha_{4}}}=-x_{A_{1} 1} \sum_{\Gamma \beta} \Gamma_{\Lambda_{1} \alpha_{1} \Gamma \beta}^{0} \begin{array}{c}
V_{A_{1} A_{2} A_{3} A_{4}} \\
\Gamma \Lambda_{2} \Lambda_{3} \Lambda_{4} \\
\beta \alpha_{2} \alpha_{3} \alpha_{4}
\end{array}
\end{aligned}
$$

and $V$ on the right-hand side of (5.8) is defined by

$$
\begin{array}{cc}
U_{A_{1} A_{2} A_{3} A_{4}}=x_{A_{1} i_{1}} & V_{A_{1} A_{2} A_{3} A_{4}} . \\
i_{1} i_{2} i_{3} i_{4} & \Lambda_{1} \Lambda_{2} \Lambda_{3} \Lambda_{4} \\
\Lambda_{1} \Lambda_{2} \Lambda_{3} \Lambda_{4} & \alpha_{1} \alpha_{2} \alpha_{3} \alpha_{4} \\
\alpha_{1} \alpha_{2} \alpha_{3} \alpha_{4} &
\end{array}
$$

Finally $\sum_{\left(y_{2} y_{3} y_{4}\right)}$ means the sum over all cyclic permutations of $y_{2}, y_{3}$, and $y_{4}$, i.e.

$$
\sum_{\left(y_{2} y_{3} y_{4}\right)} A_{y_{2} y_{3} y_{4}}=A_{y_{2} y_{3} y_{4}}+A_{y_{4} y_{2} y_{3}}+A_{y_{3} y_{4} y_{2}} \text {. }
$$

Because equations (5.3) do not contain derivatives with respect to time we set $t=0$ :

$$
\varphi_{Z_{1} Z_{2}}\left(\vec{x}_{1}, \vec{x}_{2}\right)=\varphi_{Z_{1} Z_{2}}\left(0, \vec{x}_{1}, 0, \vec{x}_{2}\right) .
$$

[1] UA 1 Collaboration, Phys. Lett. 122 B, 103 (1983).

[2] H. Georgi and S. L. Glashow, Phys. Rev. Lett. 32, 438 (1974). For a recent review see P. Langacker, Phys. Rep. 72 c, 185 (1981).

[3] E. Gildener, Phys. Lett. 92 B, 111 (1980).

[4] E. Witten, Nucl. Phys. B 185, 513 (1981).

[5] For a recent review see e.g. R. D. Peccei, Composite models of quarks and leptons, Lectures presented at the Arctic School of Physics "Gauge Theories of the Eighties", Akäslompolo, Finland, August 1982.

[6] H. Harari, Phys. Lett. 86 B, 83 (1979); M. A. Shupe Phys. Lett. 86 B, 87 (1979).

[7] H. Harari and N. Seiberg, Phys. Lett. 98 B, 269 (1981); H. Harari and N. Seiberg, Nucl. Phys. B 204, 141 (1982).

[8] M. Gell-Mann, "The eightfold way: a theory of strong interaction symmetry", Caltech Report CT SL-20 (1961), reprinted in "The eightfold way", ed. M. GellMann and Y. Ne'eman, W. A. Benjamin.

[9] D. Großer and T. Lauxmann, J. Phys. G: Nucl. Phys. 8, 1505 (1982).

[10] F. Bopp, Ann. Phys. (Germany) 38, 345 (1940); B. Podolski, Phys. Rev. 62, 68 (1942); A. Pais and G. E. Uhlenbeck, Phys. Rev. 79, 145 (1950); K. Wildermuth, Z. Naturforsch. 5a, 373 (1950).

[11] H. Stumpf, Z. Naturforsch. 36 a, 1289 (1981).

[12] A. Zee, Phys. Lett. 109 B, 183 (1982); M. Kaku, Nucl. Phys. B 203, 285 (1982).

[13] S. Ferrara and B. Zumino, Nucl. Phys. B 134, 301 (1978).

[14] T. D. Lee and G. C. Wick, Phys. Rev. D2, 1033 (1970).

[15] H. Stumpf, Z. Naturforsch. 36 a, 785 (1981).

[16] H. Stumpf, in preparation.

[17] K Symanzik, in "Fundamental Interactions at High Energies II", ed. A. Perlmutter, R. W. Williams, and G. J. Iverson, Gordon and Breach, New York 1970.
Since we interpret $\vec{x}_{1}$ and $\vec{x}_{2}$ as the coordinates of the constituent rishons we require that our amplitudes are square integrable with respect to $\vec{x}_{2}-\vec{x}_{1}$. This defines our eigenvalue problem. The amplitude $\varphi_{Z_{1} Z_{2}}$ is a bispinor. Hence to find the amplitudes for scalar and vector bosons we have to decompose this reducible representation into its irreducible components. The resulting equations are still quite complicated and a rigorous solution may not be possible. However, since they are of the same type as the Schrödinger equation of nonrelativistic quantum mechanics, they should be amenable to variational methods. This will be treated in forthcoming publications for rishon-antirishon bound states and for three rishon bound states which are to be interpreted as leptons and quarks.

\section{Acknowledgements}

We are grateful to the Deutsche Forschungsgemeinschaft for financial support.

[18] D. Maison and H. Stumpf, Z. Naturforsch. 21 a, 1829 (1966).

[19] I. Tamm, J. Phys. USSR 9, 449 (1945); S. M. Dancoff, Phys. Rev. 78, 382 (1950); F. J. Dyson, Phys. Rev. 90, 994 (1953); W. Zimmermann, Nuovo Cim. Suppl. 11, 43 (1954).

[20] A. Friederich, W. Gerling, and K. Bleuler, Z. Naturforsch. 30a, 142 (1975); K. Bleuler, A. Friederich, H. R. Petry, and D. Schütte, in "Quanten und Felder”, ed. H. P. Dürr, Vieweg, Braunschweig 1971.

[21] A. Chodos, R. L. Jaffe, K. Johnson, C. B. Thorn, and V. F. Weisskopf, Phys. Rev. D9, 3471 (1974); see also F. Close, An Introduction to Quarks and Partons, Academic Press, London.

[22] K. G. Wilson, Phys. Rev. D 10, 2445 (1974); for a review see J. M. Drouffe and C. Itzykson, Phys. Rep. C38, 133 (1978).

[23] H. A. Bethe and E. E. Salpeter, Phys. Rev. 82, 309; (1951); M. Gell-Mann and F. Low, Phys. Rev. 84, 350 (1951).

[24] P. Hasenfratz and I. Montvay, Phys. Rev. Lett. 50, 309 (1983).

[25] N. Nakanishi. Phys. Rev. 138 B, 1182 (1965)

[26] H. Terazawa, Phys. Rev. D 22, 184 (1980); K. Akama, Prog. Theor. Phys. 64, 1494 (1980).

[27] D. Amati and G. Veneziano, Nucl. Phys. B 204, 451 (1982).

[28] H. P. Dürr, in "Unified Theories of Elementary Particles", ed. P. Breitenlohner and H. P. Dürr, Springer, Berlin.

[29] W. Heisenberg, Einführung in die einheitliche Feldtheorie der Elementarteilchen, S. Hirzel Verlag, Stuttgart.

[30] H. Stumpf, Z. Naturforsch. 37 a, 1295 (1982).

[31] H. Stumpf, K. Scheerer, and H. G. Märtl, Z. Naturforsch. 25a, 1556 (1970). 